\title{
Generation of frequency combs and dissipative solitons in integrated microresonators in self- injection locking regime
}

\author{
Sofya Agafonova ${ }^{1,2, *}$, Andrey Voloshin ${ }^{1}$, Alexander Gorodnitskiy ${ }^{1,2}$, Artem Shitikov ${ }^{1,3}$, \\ Valery Lobanov ${ }^{1}$, and Michael Gorodetsky ${ }^{1,3}$ \\ ${ }^{1}$ Russian Quantum Center, 112205 Moscow, Russia \\ ${ }^{2}$ Moscow Institute of Physics and Technology, 141701 Dolgoprudny, Moscow region, Russia \\ ${ }^{3}$ Moscow State University, Faculty of Physics, 119991 Moscow, Russia
}

\begin{abstract}
We developed a method to generate frequency combs that implements compact multimode diode laser. This technique allows for generating of low-noise frequency combs with the linewidth around $100 \mathrm{kHz}$ due to self-injection locking effect.
\end{abstract}

\section{Introduction}

A frequency comb is a set of equidistant narrow lines in the ultraviolet, visible or infrared regions of the frequency space. It is used in spectroscopy, calibration of astrophysical spectrometers, and precision measurements. In recent years, the generation of optical combs in microcavities with whispering gallery modes has been actively studied [1]. Keeping the characteristics of modern precision instruments for optical measurements, the technique allows for a significant reduction in the size of a generator.

\section{Motivation}

Modern methods for generation of frequency combs in microresonators use powerful singlefrequency narrow lasers. Their size is several orders of magnitude higher than the sizes of microresonators, which prevents the actual decrease in the size of a generator.

We have developed a method for generating optical combs using a compact multi-mode diode laser. The self-injection locking effect on an integrated microcavity [2] allows to transform the initial spectrum with many lines with a width of the order of ten megahertz into one line with a width of about $100 \mathrm{kHz}$. The resulting source is then used to generate a lownoise optical comb in the same microcavity $[3,4]$. A similar result was obtained in a crystal resonator with whispering gallery modes [5].

\footnotetext{
*Corresponding author: sofya.agafonova@gmail.com
} 


\section{Setup}

We used a multimode diode laser with a spectral width of about $1 \mathrm{THz}$ and a central wavelength of about $1535 \mathrm{~nm}$. It was placed in front of one end of the microcavity bus guide, and in front of the other end there was a lensed fiber collecting the transmitted light. Tuning to one of the modes of the integrated microresonator described in [6] by changing the current and temperature of the diode leads to a narrowing of the spectrum to one line with a width of about $100 \mathrm{kHz}$. Inside the same mode, we achieved the generation of a frequency comb with a spectral width of about $400 \mathrm{~nm}$. We observed several comb generation regimes, among which there is one with an optical spectrum with a smooth envelope described by the function

$$
\psi(\omega)=p_{1} \operatorname{sech}^{2}\left(\left(\omega-p_{2}\right) / p_{3}\right)
$$

and low radio wave noise (see Fig. 1). Such a configuration indicates a soliton state [7], which is extremely important for many applications.

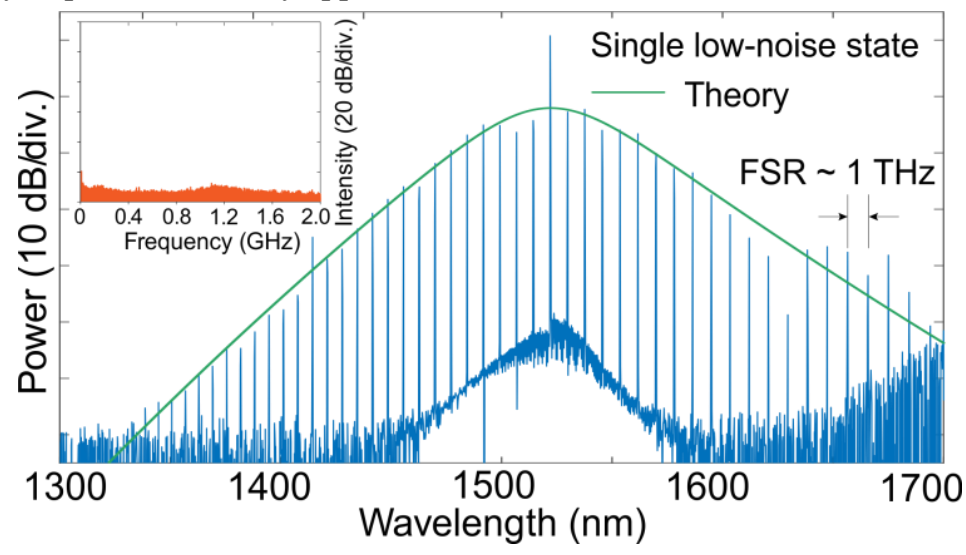

Fig. 1. The spectrum of the frequency comb in self-injection locking regime.

The study was funded by a grant from the Russian Science Foundation (project No. 17-12-01413).

\section{References}

1. P. Del'Haye, A. Schliesser, O. Arcizet, T. Wilken, R. Holzwarth, T. J. Kippenberg, Nature, 450 (2007)

2. N. M. Kondratiev, V. E. Lobanov, A. V. Cherenkov, A. S. Voloshin, N. G. Pavlov, S. Koptyaev, M. L. Gorodetsky, OSA Pub., 25 (2017)

3. A. S. Raja, A. S. Voloshin, H. Guo, S. E. Agafonova, J. Liu, A. S. Gorodnitskiy, M. Karpov, N. G. Pavlov, E. Lucas, R. R. Galiev, A. E. Shitikov, J. D. Jost, M. L. Gorodetsky, T. J. Kippenberg, Nat. Com., 10 (2019)

4. S. Agafonova, A. Voloshin, A. Gorodnitskiy, A. Shitikov, M. Pfeiffer, T. Kippenberg, M. Gorodetsky, ICLO (2018)

5. N. G. Pavlov, S. Koptyaev, G. V. Lihachev, A. S. Voloshin, A. S. Gorodnitskiy, M. V. Ryabko, S. V. Polonsky, M. L. Gorodetsky, Nat. Phot., 12 (2018)

6. M. H. P. Pfeiffer, C. Herkommer, J. Liu, H. Guo, M. Karpov, E. Lucas, M. Zervas, T. J. Kippenberg, Optica, 4 (2017)

7. T. Herr, V. Brasch, J. D. Jost, C. Y. Wang, N. M. Kondratiev, M. L. Gorodetsky, T. J. Kippenberg, Nat. Phot. , 8 (2014) 